\title{
The use of the Appendix for Reconstruction of the Left Ureter: A Case Report
}

Hussein Soliman*

Surgical unit, National Cancer Institute, Cairo University, Egypt

\begin{abstract}
We present the case of a 38-year-old female with a locally advanced recto-sigmoid adenocarcinoma. Preoperative CT scan showed that the tumour is closely related to the left psoas muscle, with total encasement of the left ureter, causing Grade II left sided hydronephrosis. Exploration was done, and an extended left hemicolectomy, with en-bloc resection of a segment of about $6 \mathrm{~cm}$ from the left ureter was performed. After trying to approximate both cut ends, it was clear from the start that they will not reach, and a bridge would be needed to reconstruct the ureter.
\end{abstract}

Keywords: Appendix; Reconstruction; Left ureter; Hemicolectomy; Hemostasis; Adenocarcinoma

\section{Introduction}

The typical way we would previously repair this defect was with an ileal loop [1] interposition between both ends, however I thought that would be quite aggressive, and was looking around for a simpler option. After careful inspection of the abdomen, I noticed the patient to have a relatively long appendix [2], but more importantly, a long mesoappendix. After testing to see if the appendix would reach, and it appeared it would, the decision was taken. We will use the appendix to reconstruct the left ureter [3]. The appendicular base was ligated and divided from the caecum, and the mesoappendix was stretched, with meticulous dissection and care not to injure or harm the ever so sensitive appendicular artery and vein, reaching the area in question. The appendicular tip was the cut-off, transforming it into a pedicled tube, almost the exact same size and diameter as the ureter. After insertion of a double-J ureteric catheter, the end-to-end anastomosis between the appendix and the ureter was performed using PDS 4/0 sutures. Between 4-5 interrupted sutures were used for each of the 2 ends. A single layer of vicryl 3/0 interrupted sutures was used to suture the colon, and 2 tube drains were placed, and the abdomen was closed after haemostasis [4].

\section{Case Study}

We present the case of a 38-year-old female with a locally advanced sigmoid adenocarcinoma that complained from repeated attacks of bleeding per rectum through the course of two months [5].

An abdominopelvic US done revealed left mild hydronephrosis with dilated upper segment of the left ureter, and a left ovarian simple cyst, measuring $3 \times 2.5 \mathrm{~cm}$. Further investigation with a CT of the abdomen and pelvis with oral and IV contrast was done. Findings of the CT were a long segment of tumoral wall thickening involving the distal part of the descending colon and the proximal part of the sigmoid colon, measuring approximately $87 \mathrm{~mm}$ in length, and grade II hydronephrosis of the left kidney. The tumor invaded beyond the serosa of the colon into the left psoas major muscle and the left ureter (Figure 1). This entailed colonoscopy examination, which revealed a large polypoidal lesion in the distal descending colon, of which multiple biopsies were taken. Histopathological examination of the biopsies revealed ulceration and chronic inflammation, but could not rule out malignancy, due to the presence of an ulcerated large polypoidal mass [6]. Preoperatively, a complete blood count, coagulation profile, liver functions and $U \& E$ were done, all of which were within normal ranges.
The patient was explored through a midline laparotomy. Upon exploration, a mass was found in the distal descending colon, invading a segment of the left ureter of about 5 centimeters -at about the junction between its proximal two-thirds and distal one-third- and the adjacent part of the left psoas major muscle, together with the left ovary. Operation proceeded in the form of left hemicolectomy [7], en bloc with the invaded segment of the left ureter and the adjacent psoas muscle, and left salpingo-oophorectomy. End-to-end colo-colic primary anastomosis was performed to restore bowel continuity, using continuous $3 / 0$ vicryl sutures for the first layer, reinforced by a second layer of interrupted inverting Lambert's sutures.

The next important step was addressing the resultant ureteric defect, which was about 6 centimeters. It was clear intraoperative that a primary anastomosis would not be feasible. Traditionally, an ileal loop interposition graft would be used as a conduit to repair such a defect. However, the patient had a relatively long appendix and mesoappendix (Figure 2), and so a decision to use the appendix for ureteric reconstruction was taken. The base of the appendix was separated from the cecum, and the stump buried using the regular vicryl $3 / 0$ pursestring suture, as in normal appendectomy operations [8]. The appendix was left attached to the mesoappendix to preserve its blood supply through the appendicular artery and vein, and mobilization of the proximal part of the ascending colon was done, transposing the cecum with the appendix towards the left ureter. The appendicular tip was then cut off, transforming it into a pedicled tube, almost the exact same size and diameter as the ureter. A cannula was inserted in its lumen, and careful and repeated irrigation was performed using normal saline. Anastomosing the end that corresponds to the appendicular tip to the proximal end of the ureter -as it anatomically should- was not settling well, since the end corresponding to the base of the appendix would not reach the distal ureteric segment in the pelvis. So, the appendix was flipped -with care not to twist its pedicle- in such a manner, that the tip

*Corresponding author: Soliman H, Associate Professor, Surgical unit, National Cancer Institute, Cairo University, Egypt, Tel: +201208285664; E-mail soliman@daad-alumni.de

Received July 20, 2017; Accepted May 18, 2018; Published May 21, 2018

Citation: Soliman H (2018) The use of the Appendix for Reconstruction of the Left Ureter: A Case Report. J Cancer Sci Ther 10: 135-139. doi: 10.4172/19485956.1000530

Copyright: (C) 2018 Soliman H. This is an open-access article distributed unde the terms of the Creative Commons Attribution License, which permits unrestricted use, distribution, and reproduction in any medium, provided the original author and source are credited. 
Citation: Soliman H (2018) The use of the Appendix for Reconstruction of the Left Ureter: A Case Report. J Cancer Sci Ther 10: 135-139. doi: 10.4172/1948-5956.1000530

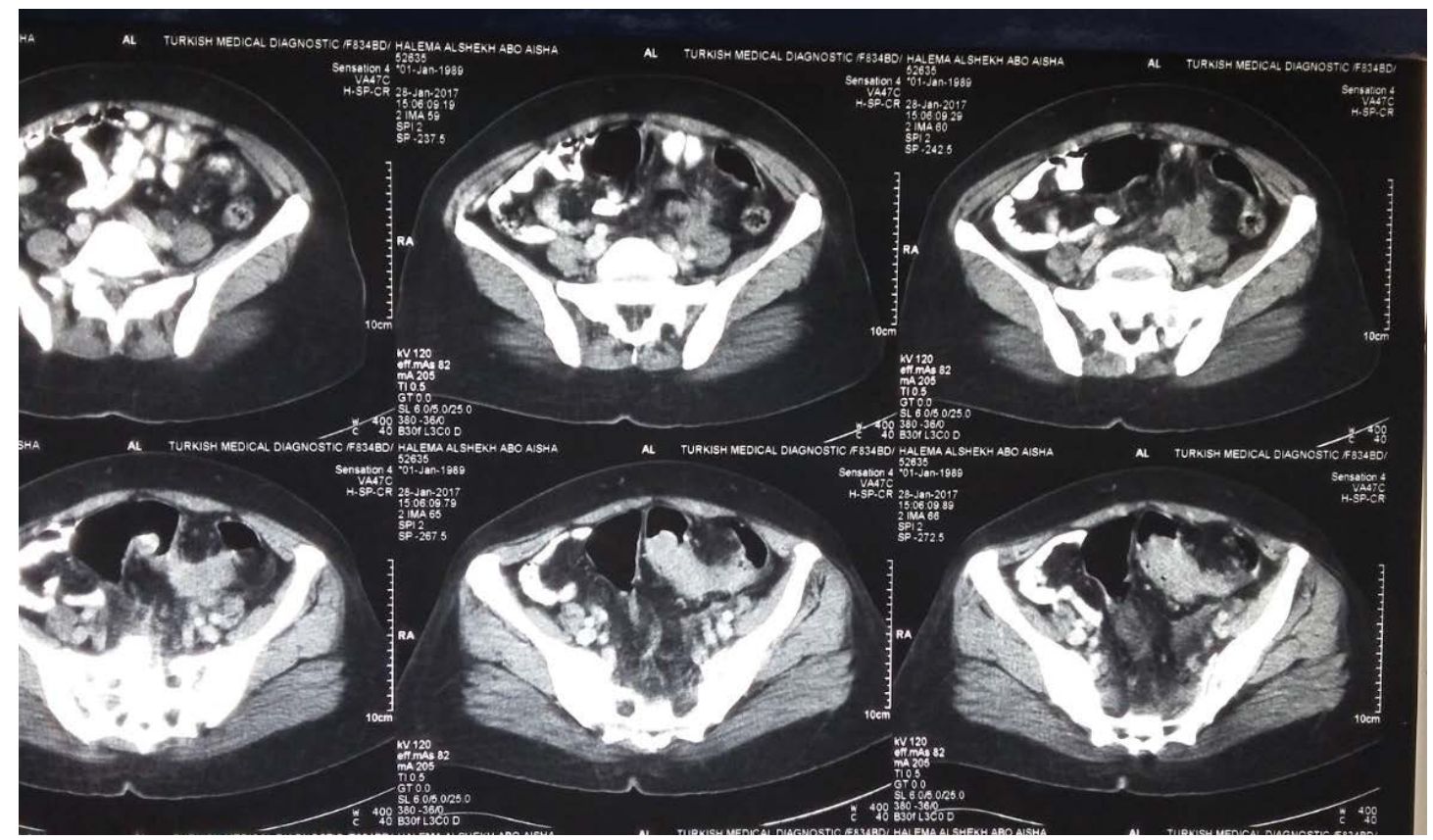

Figure 1: CT showing local extension of the tumor.

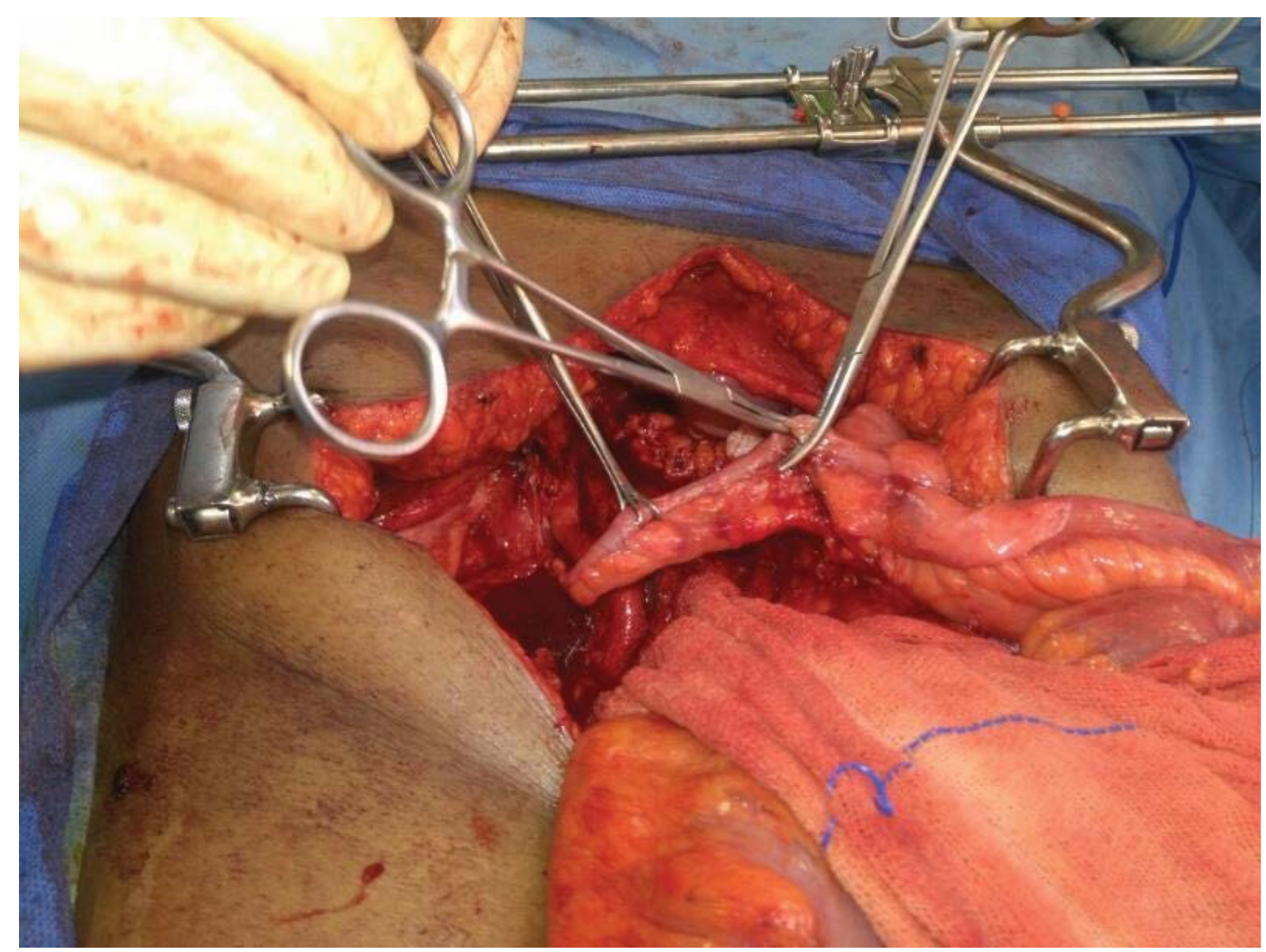

Figure 2: Intra-operative picture of long appendix and mesoappendix.

would be reaching down, thus making it easier to anastomose it to the distal ureteric segment, and the base of the appendix would be directed upwards to anastomose it to the proximal ureteric segment (Figure 3). After making sure that the blood supply was still intact, and the 
Citation: Soliman H (2018) The use of the Appendix for Reconstruction of the Left Ureter: A Case Report. J Cancer Sci Ther 10: 135-139. doi: 10.4172/1948-5956.1000530

appendix was still viable, with no compromise of its blood supply - which was facilitated by the cecal mobilization, the anastomosis was begun. A 6/26 double-J ureteric catheter [9] was inserted into the proximal ureteric segment, and through the reversed appendix, into the distal ureteric segment, to avoid postoperative stricture in the anastomotic sites (Figure 4). The appendix was then anastomosed to the ureter, end-to-end on both sides, using 5 interrupted PDS 5/0 sutures for each end. Hemostasis was ensured, and two 18-Fr tube drains were inserted, and the abdomen was then closed in layers.

The patient passed a very smooth post-operative course and was discharged on the $5^{\text {th }}$ post-operative day in good general health. We did an angiography, and a CT urography [10] about 2 weeks after, to make sure that there were no problems encountered, and all the routine postoperative images and blood works were in order (Figure 5).

\section{Results}

The results of ureteral replacement with ileum are difficult to assess because this form of surgery is not standardized, and patient selection varies considerably between the different series. Furthermore, the objective goals of the procedure are not clearly defined. The values of BUN or serum creatinine that have been reported in some series are probably not sufficient to define the outcome in patients with bilateral kidneys. Dilation of the upper urinary tract is another parameter used in the literature. However, it is difficult to discriminate between persistent dilation despite reduction of ureteral obstruction and those cases in which dilation persists as a result of obstruction and/or reflux after surgery. To date, no reports including diuretic isotope scintigraphy have been published.

\section{Discussion and Conclusion}

Partial or complete ureteral replacement remains a challenge for the urologist. The maintenance of kidney function is the primary goal of this procedure. The crucial problem with ureteral replacement is that in most cases several surgical procedures and frequently radiotherapy have been performed beforehand, and kidney function is usually already impaired at the time of intervention. Therefore, standardized recommendations may not be applicable, and the approach must be tailored according to the individual situation.

Several ureteral substitutes have been proposed, including blood vessels, fallopian tubes, and the appendix. Nevertheless, all these tissues or organs and the corresponding procedures are correlated with a significant morbidity, including recurrent infection, stone, or stricture formation, with a worsening of kidney function in many patients. Because larger series have rarely been reported, the results and morbidity of the various procedures differ significantly.

In the few studies with long-term results, a favorable outcome has been reported in up to $85 \%$ of the cases. This does not include patients with impaired renal function with serum creatinine levels greater than $2.0 \mathrm{mg} / \mathrm{dl}$. In this population, fewer than $50 \%$ will benefit from ureteral replacement. In summary, ureteral replacement with ileum is a feasible technique that carries considerable perioperative and longterm morbidity and should therefore be considered only as a secondline treatment in selected patients.

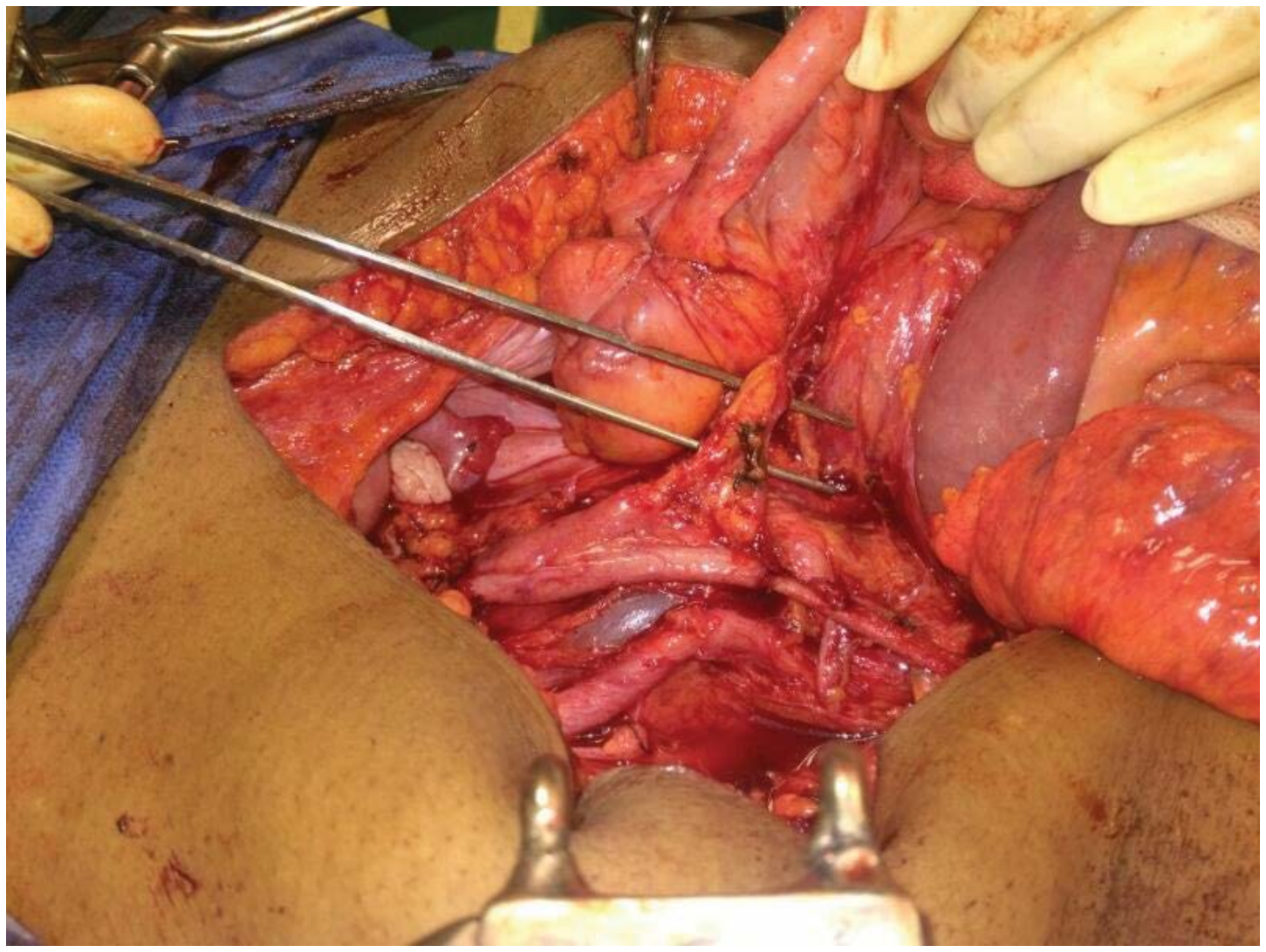

Figure 3: Making sure that the pedicle hasn't been compromised from the twist. 


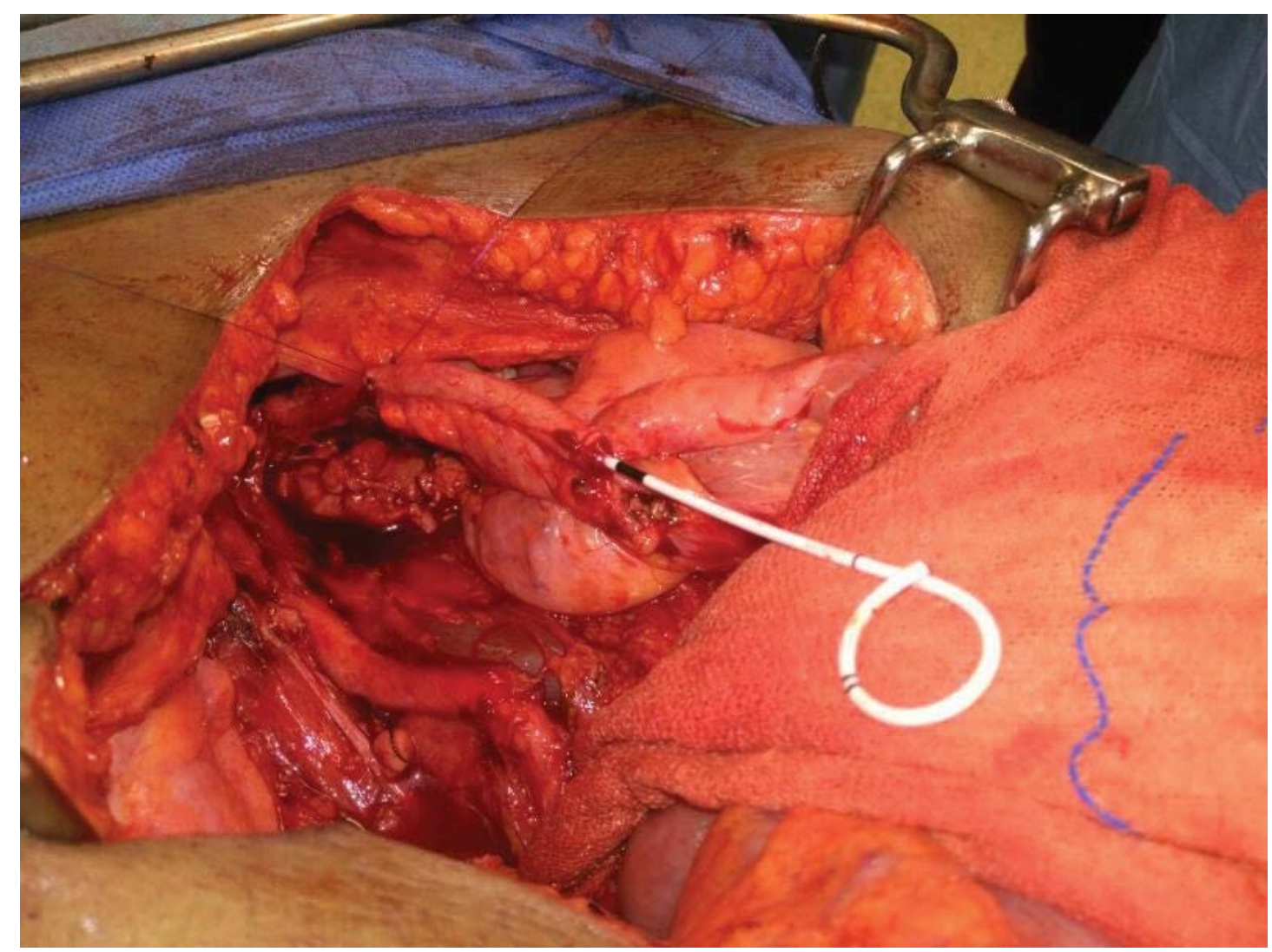

Figure 4: Insertion of the double $\mathrm{J}$ catheter.

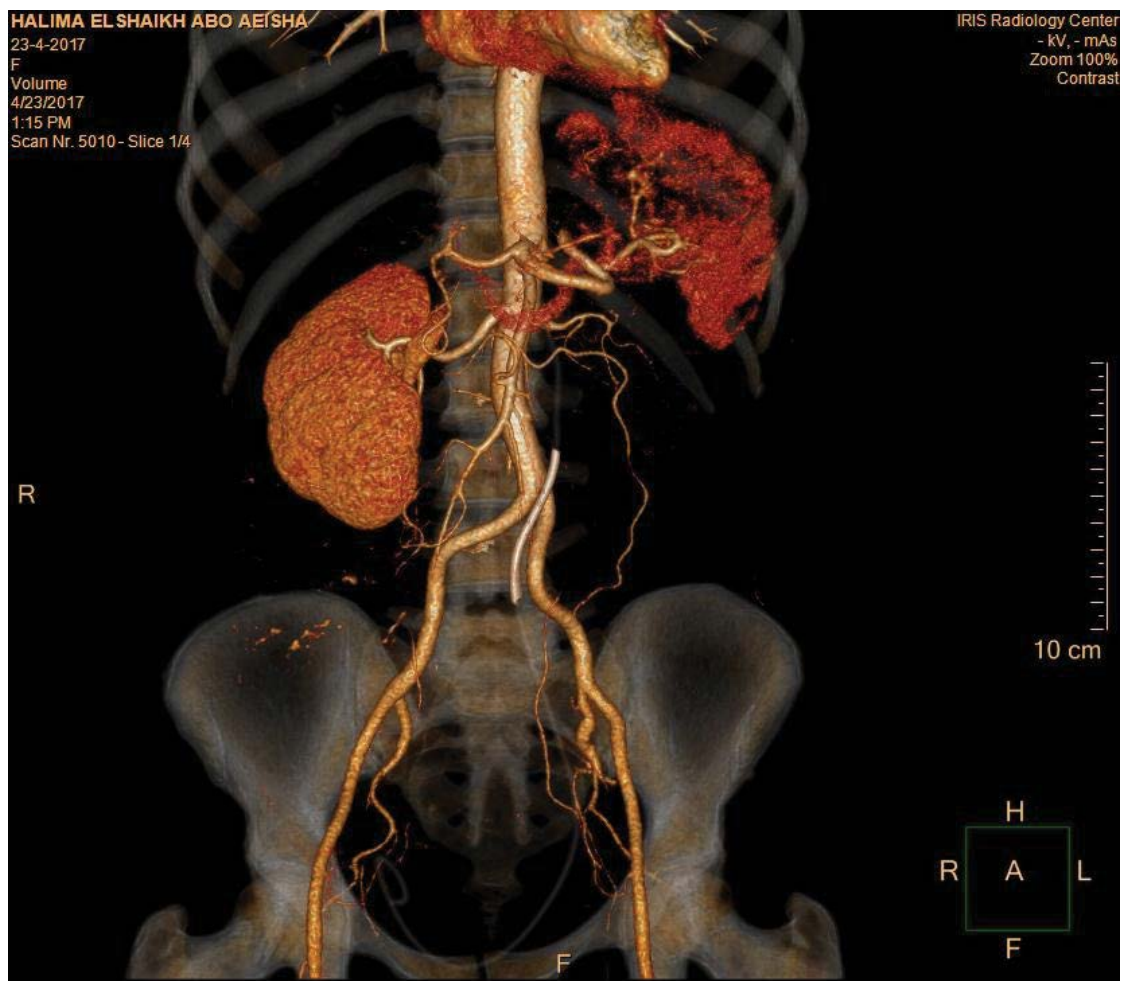

Figure 5: CT angiography showing appendicular artery diverging to the left, with the appendix clearly showing, and the double $\mathrm{J}$ in place. 
Citation: Soliman H (2018) The use of the Appendix for Reconstruction of the Left Ureter: A Case Report. J Cancer Sci Ther 10: 135-139. doi: $10.4172 / 1948-5956.1000530$

\section{References}

1. Samples J, Evans K, Chaumont N, Strassle P, Sadiq T, et al. (2017) Variant two-stage ileal pouch-anal anastomosis: An innovative and effective alternative to standard resection in ulcerative colitis. J Am Coll Surg 224: 557-563.

2. Bednarczuk T, Bolanowski M, Zemczak A, Bałdys-Waligórska A, BlicharzDorniak J, et al. (2017) Neuroendocrine neoplasms of the small intestine and appendix-management guidelines (recommended by the Polish Network of Neuroendocrine Tumours). Endokrynol Pol 68: 223-236.

3. Hayashi Y, Kawahara T, Hattori Y, Shimokihara K, Tsutsumi S, et al. (2017) Xantho-granulomatous pyelonephritis with incomplete double ureter. Case Rep Med 2017: 2392670

4. Palagina IA, Kudria MY, Lalymenko OS (2016) Role of nitric oxide metabolites within the impact of the sub-toxic succinamides doses on state of hemostasis. Fiziol Zh 62: 34-42.

5. Helsloot L, Reynders D, Cools P, Wauters J (2017) Adenocarcinoma of the Rectum in a 27-year-old Patient with Usher Syndrome: Is there a genetic correlation? Acta Gastroenterol Belg 80: 77-78.

6. Kakkad K, Padhi T, Pradhan K, Agrawal KC (2016) A study of clinical, bacteriological and histopathological correlation in leprosy cases attending a government medical college in western Odisha: Some observations. Indian J Lepr 88: 97-103.

7. Ćwiertnia G, Dyaczyński M (2017) Right hemicolectomy under spinal anaesthesia due to cancer of ascending large bowel-case report. Wiad Lek 70: 1013-1015.

8. Hall NJ, Eaton S, Abbo O, Arnaud AP, Beaudin M, et al. (2017) Appendectomy versus non-operative treatment for acute uncomplicated appendicitis in children: Study protocol for a multicentre, open-label, non-inferiority, randomised controlled trial. BMJ Paediatr Open 18: 1.

9. Dejmek M, Kučera T, Ryšková L, Čermáková E, Šponer P (2017) Bacteriuria and symptomatic urinary tract infections during antimicrobial prophylaxis in patients with short-term urinary catheters-prospective randomised study in patients after joint replacement surgery. Acta Chir Orthop Traumatol Cech 84 368-371.

10. Bombiński P, Brzewski M, Warchoł S, Gołebiowski M (2017) One-phase splitbolus CT urography: A novel approach to reduce radiation dose in diagnostics of congenital anomalies of kidneys and urinary tract in children. Dev Period Med 21: 402-407. 